\title{
Wireless Communication Solution for Distributed Structural Health Monitoring
}

\author{
Artur Andrzejczak, Paweł Łęczycki, Maciej Makowski, Bartosz Pękosławski, Piotr Pietrzak, \\ and Andrzej Napieralski
}

\begin{abstract}
This paper describes a design of wireless distributed SHM (Structure Health Monitoring) system with a particular emphasis on comparison of wireless communication standards. The presented solution is being deployed in the TULCOEMPA project. Several wireless communication standards are compared, with their benefits, disadvantages and typical areas of application. A choice of proper ISM (Industrial Scientific Medical) band and reasons for use of Wireless Sensor Networks are also discussed. The last part of this paper presents the proposed structure and designed prototype. The chosen architecture of the system and the program algorithm used for communication and measurements are described.
\end{abstract}

Keywords-wireless sensor networks, IEEE 802.15.4, Bluetooth low energy, DASH7, IEEE 802.11n, ZigBee, weightless, structural health monitoring

\section{INTRODUCTION}

A DISTRIBUTED Structural Health Monitoring makes it possible to asses a condition of civil engineering structures, such as bridges, halls, dams, etc. As technology in civil engineering evolves, the above objects become more complex, therefore it is more difficult to sustain high reliability. Civil structures are exposed to extensive loadings, which might lead to material degradation, that may result in a collapse of an object. Loadings can be caused by environmental conditions such as strong wind, snowfalls, or earthquakes. When concerning bridges, long term overload might also lead to significant damage. One of possible implementations of SHM is to measure object strain in selected points, together with environmental conditions, such as humidity and temperature. Processed, by proper algorithms, data results with information about health of monitored object are sent to persons responsible for structure condition. In the case of alarming results, corresponding maintenance measures can be applied to avoid risk of damage, and accordingly might save human lives.

There are many situations, when it is worth to consider the wireless communication for distributed SHM sensors. The most significant advantage is avoidance of structural cabling. In particular cases, providing wire connection between measurement nodes comes with unacceptable costs. Obstacles can be both physical or formal. In the first case, high temperature or humidity may lead to faster degradation of insulation and connectors. These conditions can cause higher costs, which are also related to installation procedure and proper wire quality. Formal obstacles often come with additional time

The authors are with the Department of Microelectronics and Computer Science of Łódź University of Technology, Poland (e-mail: andrzejczak@dmcs.pl). and expenses. The above issues become increasingly important when measurement system is being installed for short, specified amount of time, usually for diagnostics purposes. In this kind of situations, despite power line availability, wireless communication is still an efficient solution. This paper discusses choice of a proper wireless communication standard for strain measurement system, which is being designed for the TULCOEMPA project.

The TULCOEMPA project is based on the Swiss-Polish cooperation between the Technical University of Lodz (DMCS, $\mathrm{KBB}^{1}$ and EMPA ${ }^{2}$. Multipurpose program of the project covers civil engineering and structural health monitoring of buildings. Unanchored prestressed carbon fiber reinforced polymer laminates will be used for bridge strengthening. To ensure effectiveness of this solution, a long term monitoring of the structure will be applied [1].

\section{WiRELESS COMMUNICATION SOLUTION}

In the TULCOEMPA project, measurements will cover both static and dynamic effects on the object. In other words, strain will be measured with low frequency, and additionally, with high frequency for short periods of time. This will allow to asses behaviour of the bridge during heavy loadings. Dynamic measurements will occur only when presence of a heavy vehicle is detected by TRS (Truck Recognition System). It will allow to enable a reduction of WSS (Wireless Strain Sensor) devices power consumption. The WSS nodes are deployed on the bridge (Fig. 1).

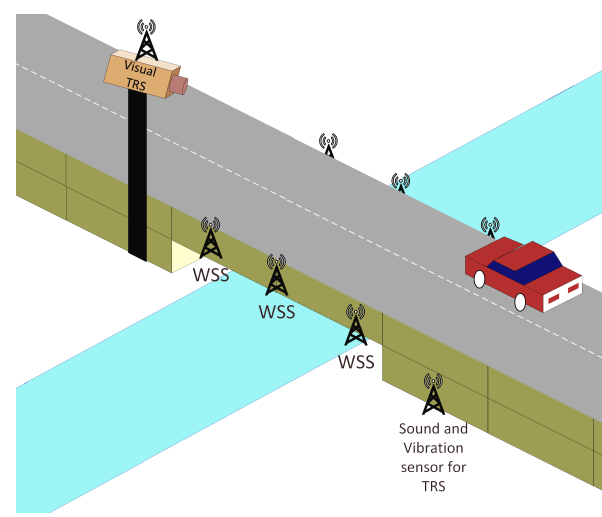

Fig. 1. TRS with WSS deployment on the bridge.

\footnotetext{
${ }^{1}$ Katedra Budownictwa Betonowego, Lodz University of Technology

${ }^{2}$ Swiss Federal Laboratories for Materials Science and Technology (Eidgenssische Materialprfungs und Forschungsanstalt)
} 
Furthermore, environmental conditions (temperature and humidity) which might also have an influence on the bridge, will be monitored by WSS. The system deployed on the object will use wireless communication, therefore a proper standard must be chosen. Trigger signal for enabling dynamic measurements will be provided by TRS, which uses optical, vibration, electromagnetic, and acoustic sensors to detect presence of a truck on the bridge. TRS is not discussed in this paper.

\section{A. ISM BANDS}

As most of currently applied wireless communication solutions, WSS devices will use the ISM band. It is a set of bands, which does not require any licence or permission to use with a limited transmit power. Primarily, ISM frequencies were intended for industrial, scientific and medical purposes, other than communication, for example, microwave ovens. Nowadays, in spite of original destination, ISM is generally used for short range radio communication, often accompanied by creation of small network infrastructures. Due to initial use and wide applicability of this kind of equipment, transceivers working in ISM band should be able to deal with various interferences, whose amounts depends on selected band. The selection of frequency should also depend on achievable range, which has impact on energy consumption and node deployment density.

The distance over which connection can be set up depends on frequency, at which transceiver operates. When antennas have no visual contact, communication takes place through the obstacles, reflecting from them and bending. What is more, attenuation of materials depends on a frequency of an electromagnetic wave passing through it. Attenuation is decreasing with a wavelength. It is caused by resonance of particles in the atmosphere, mainly water and oxygen. As a result of this phenomenon, range decreases. Another relevant effect is a reflection of an electromagnetic wave. Similarly to the attenuation, the higher is frequency of a wave, the more energy is absorbed by an object. Therefore energy reflected from an obstacle is reduced and as a result range is decreased. Next phenomenon is diffraction. When a wave encounters an obstacle, it bends around it. The angle of deflection depends on the length of the wave and the relative size of the object. The lower frequency, the greater deflection angle. When dealing with large obstacles, for example mountains, an electromagnetic wave with lower frequency might be more efficient with reaching node behind the obstacle. Another important issue is also the amount of interferences in a selected band. This is a crucial issue in 2.4-GHz band, since it is used by many ISM devices, like microwaves, WiFi networks, Bluetooth adapters, analogue surveillance cameras, etc. Accumulation of significant number of mentioned devices in a limited space, causes interference, which can lead to decreased network coverage and capacity and as a result more energy for communication is required. Presented factors make $430-\mathrm{MHz}$ band an interesting choice. However, the $860-\mathrm{MHz}$ one can be better selection because of its lower popularity, hence, a decreased chance for interference.

\section{B. Wireless Sensor Networks}

Wireless Sensor Networks make it possible to use multiple strain measurement devices deployed on monitored structure. Control of several strain points enables better assessment of object condition. What is more, along with progressive state changes, locations of measurement points can be changed, which might be troublesome for solutions with structural cabling or an inflexible wireless network.

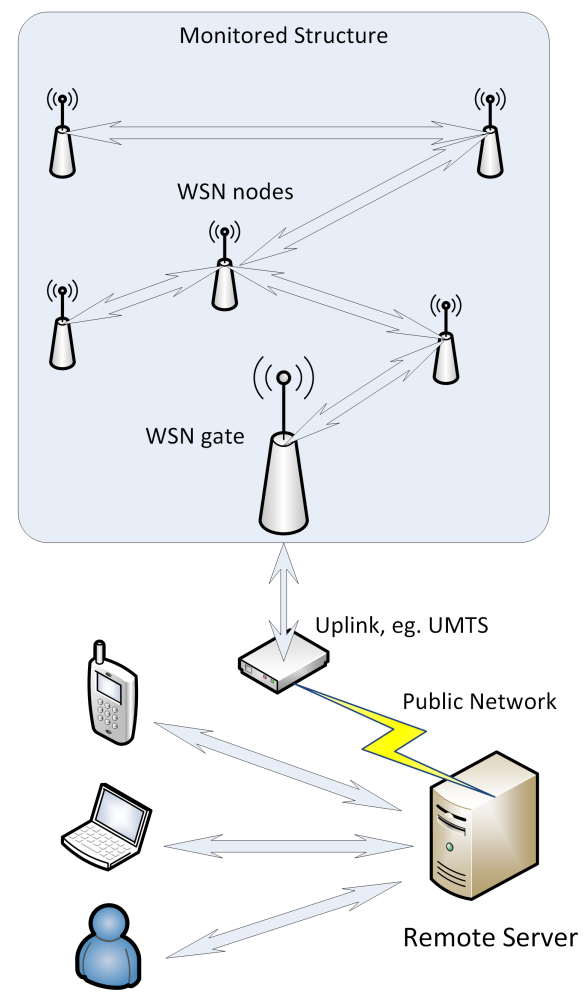

Fig. 2. Wireless sensor network structure.

Wireless Sensor Networks consist of a number of small devices, equipped with transceivers, microcontrollers and energy sources, mainly batteries. Additionally, these devices also contain sensors able to measure various physical values. Nodes can communicate wirelessly with a network coordinator, or between each other. In some cases, devices have sufficient computational resources to perform collaborative signal processing tasks. Data acquired by nodes is sent through a WSN gate (also called a sink node or network coordinator) to remote server for further processing. Structure of Wireless Sensor Network for strain measurement in the TULCOEMPA project is presented in Fig. 2. In many Wireless Sensor Networks it is difficult to replace node batteries, therefore efficient power management is an essential issue. When selected hardware components are not used, they should be switched off. Microcontroller supporting sleep mode with a very low power consumption is required. A transceiver should be active only when data exchange occurs. Even in an idle state, transceiver consumes significant fraction of energy, hence it is important to adjust its awake time. Transmission time can be significantly reduced by data preprocessing, compression or decision algorithms, which will determine when and what kind of results 
need to be sent forward. Computation time often costs less energy than communication, which is a key motivation in network processing [2]. Reliability of data forwarding also has a significant impact on time of transmission, and as a result, on power consumption by a wireless node. Consequently, a protocol overhead should be avoided to achieve energy efficiency. Reducing time of transceiver's transmit or receive

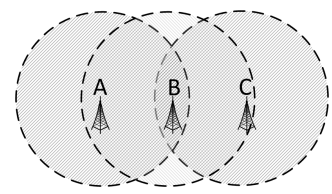

Fig. 3. Hidden node problem.

state requires time synchronization. Energy efficiency can be also increased by a proper collision avoidance mechanism. Main source of collisions in a wireless sensor network, besides the external interferences, is the Hidden Node Problem [3]. In Fig. 3 there are three nodes, A, B and C. Nodes A and B can hear each other, but node $\mathrm{A}$ and $\mathrm{C}$ cannot. In this situation, $\mathrm{C}$ might determine radio channel as free, even if $\mathrm{A}$ is already sending data to node $\mathrm{B}$. As a result, transmission from node A to B might be distorted by node C. PHY and MAC layers should provide solution for this issue. The following part of the paper presents overview of available short range wireless communication standards. It enables to asses the most efficient solution for the discussed project.

\section{802.15 .4}

IEEE 802.15.4 defines two first layers of the OSI (Open Systems Interconnection) model, physical (PHY) and medium access control (MAC). This protocol provides reliability and throughput acceptable for low-frequency measurement and control systems, while ensuring satisfactory power consumption and complexity of a transceiver. When more sophisticated communication features are required, additional higher-layer protocols like ZigBee or 6LoWPAN might be applied.

Collision avoidance mechanism is CSMA/CA, before transmission of data starts, RTS/CTS exchange occurs, which efficiently solves the Hidden Node Problem. If a source node do not receive CTS reply, it might suggest, that destination node is receiving transmission from other source at the moment. In this case, node will back-off for a specified period of time, which depends on a number of retransmission attempts and a randomly generated value. The stochastic value prevents situation, when two nodes after collision would retry transmission at the same time. During the back-off, node can be put in a sleep mode to decrease power consumption. Susceptibility to interference is also decreased by providing mechanism for upper OSI layers, which enables assessment of noise and congestion on channel. That, if required, will lead to a change of operating frequency of a whole network. Moreover, Guarantee Time Slots mechanism is provided to enhance communication reliability. For instance, when coordinator transmits a superframe with high-frequency measurement trigger, it has an unconditional access. Without GTS, communication between nodes would not be possible. The Guaranteed Time Slots allow a node to transmit frame within the time gaps of super-frame. As a result, higher layer protocols might provide QoS (Quality of Service) [3].

TABLE I

FREQUENCIES AND MODULATIONS AVAILABLE FOR IEEE 802.15.4

\begin{tabular}{|c|c|c|c|c|}
\hline Band & Number of channels & Throughput & Modulation & Area \\
\hline $868 \mathrm{MHz}$ & 1 & $\begin{array}{c}20 \mathrm{~kb} / \mathrm{s} \\
100 \mathrm{~kb} / \mathrm{s} \\
250 \mathrm{~kb} / \mathrm{s}\end{array}$ & $\begin{array}{c}\text { BPSK } \\
\text { O-QPSK } \\
\text { ASK }\end{array}$ & Europe \\
\hline $928 \mathrm{MHz}$ & 30 & $\begin{array}{l}40 \mathrm{~kb} / \mathrm{s} \\
250 \mathrm{~kb} / \mathrm{s} \\
250 \mathrm{~kb} / \mathrm{s}\end{array}$ & $\begin{array}{c}\text { BPSK } \\
\text { ASK } \\
\text { O-QPSK }\end{array}$ & North Americ \\
\hline $2.4 \mathrm{GHz}$ & 16 & $250 \mathrm{~kb} / \mathrm{s}$ & O-QPSK & World \\
\hline
\end{tabular}

There are three available frequency groups, which can be used since IEEE 802.15.4-2006[4] was published. Depending on a selected frequency and modulation, different throughput and range might be available. For instance, in $2.4 \mathrm{GHz}$ band, 16 channels are available. They are $2 \mathrm{MHz}$ wide, with $5 \mathrm{MHz}$ spacing. Details are presented in Tab. I.

IEEE 802.15.4-2011 includes additionally support for UWB (ultra-wideband) for a short range communication. Two signalling formats are available - IR (impulse radio) and CSS (chirp spread spectrum). UWB-IR provides optional feature of precision ranging using short time-domain pulses. One of three unlicensed bands can be used: $250-750 \mathrm{MHz}$ (SubGigahertz Band), 3244-4742 MHz (Low Band) and 5944$10234 \mathrm{MHz}$ (High Band). Sub-Gigahertz Band has one channel available, while Low Band - 4, and High Band - 11 . Available data rate is from $110 \mathrm{~kb} / \mathrm{s}$ up to $27.24 \mathrm{Mb} / \mathrm{s}$ ( $851 \mathrm{~kb} / \mathrm{s}$ is nominal). Throughput can be reduced in favour of available range. It is possible to increase bandwidth and as a result, achieve multipath resistance, which might improve communication range and more accurate location estimates. UWB-CSS operates only on $2.4 \mathrm{GHz}$ band with 14 channels and throughput up to $1 \mathrm{Mb} / \mathrm{s}$. Ranging is not supported. In this mode, it is difficult to detect and intercept a signal, which might be useful for specific applications [5]. A lack of commercial transceivers compliant with IEEE 802.15.4-2011 postpones applicability of UWB solution.

IEEE 802.15.4 defines two types of network devices. FFD (Full Function Device) has full protocol functionality, offering connectivity to any client of the network and as a result is able to work as a network coordinator, network node or end device. Second type is RFD (Reduced Function Device), often with decreased computing and energy resources, being able to communicate only with FFD, hence, being unable to route traffic from other nodes. IEEE 802.15.4 does not define routing. As a result, only star and peer-to-peer topologies are available. Routing can be implemented on higher OSI layers. One of the most common standards, which bases on IEEE 802.15.4, is ZigBee.

\section{ZigBee}

ZigBee has been developed by ZigBee Alliance, which associates many well-known companies from electronics industry, 
for instance Atmel, Analog Devices, Freescale, Texas Instruments and many others. ZigBee provides multi-hop topology and appropriate to its routing algorithms. It also defines ways of joining and authorizing devices into the network. Its main advantage is a support for mesh topology, which provides multiple paths from a single measurement node, through other nodes, to a sink node. It is considerable feature for sizeable networks, when not every node is in a range of WSN gate, for instance, on a larger bridge. Similarly to 802.15 .4 , on which ZigBee is based, there are three types of devices in network - coordinator, router and end device. Coordinator is a gate of the network, which allows to store and analyse acquired data on a remote server. It can also passes configuration parameters to a node. Routers are responsible for forwarding data packets from source to destination. Coordinator also has a functionality of the router, the same as some end devices, provided that they have sufficient power resources. If not, they do not participate in routing, confining themselves to end device functionality, commonly measurement. End devices communicate with router, not between another measurement nodes. Maximum number of nodes in one network is above 30 000, however a message cannot go through more than 10 nodes, which constraints size of a deployed system. Every network has its own PAN ID, which is usually randomly generated when WSN is started. Multiple networks can coexist on the same channel, although it is not an energy efficient solution due to interferences, and as a result extended back off time and numerous retransmissions. ZigBee uses IEEE 802.15.4 functionality, which enables assessment of channel interference and congestion, and if it is necessary, move whole network to other operating frequency.

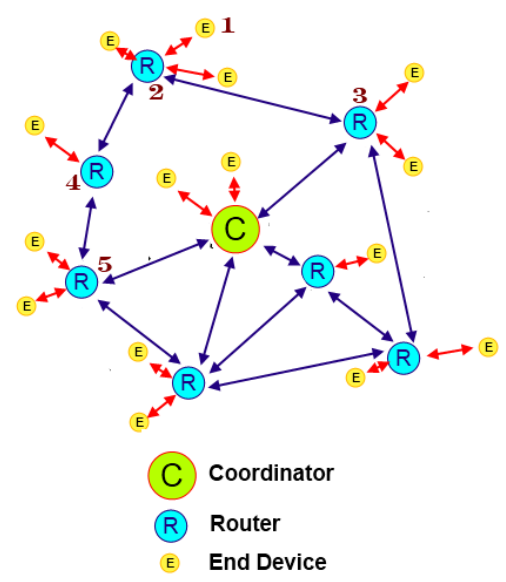

Fig. 4. Mesh routing in ZigBee.

A route of a data packet through a network depends on number of hops and the link quality between nodes in network. Moreover, route is arranged temporarily, and may change even for a reply of sent data packet. For example, in Fig. 4, when node (1) wants to send a message to coordinator, it forwards it to router (2). (2) may choose a way to coordinator, for example by node (3). If there is no connection with device (3), caused by its malfunction or interference, data can go through (4) and (5). ZigBee uses AODV (Ad Hoc On-Demand
Distance Vector) routing. Connection pathway is collated only on request. Route is determined by a sum of paths costs, which are defined by link budget between nodes. In some cases, multi-hop path can be more efficient that single-hop path with weak radio connection. Determination of route is conducted by broadcast message, which allows selected nodes to update their route table, enabling efficient data delivery in future. ZigBee Alliance also introduced ZigBee Pro, which has additional features. For instance, contrary to simpler version, transmit and return routes do not have to be the same. It is useful in situations with asymmetric link budgets. A number of hops has been increased from 10 to 30, allowing deployment of larger networks. Additionally, multicast feature has been implemented, which enables possibility of sending a message to a defined set of nodes. Enhanced features of ZigBee Pro comes with higher complexity of protocol stack and as a result, hardware requirements. The described mechanisms applied in ZigBee standard provide reliable data exchange, that is tolerant to faults and unexpected changes in network.

\section{E. 6LoWPAN}

IPv6 over Low-Power Wireless Personal Area Networks, similarly to ZigBee, is based on IEEE 802.15.4 standard, adding routing features and IP connectivity. This technology is aimed at preservation of IP networks nature in WSN, resulting cooperation with existing infrastructure. As a result, devices can be connected with other IP-based networks, without additional translation gateways. As significant advantage of 6LoWPAN over other standards is an open and freely available specification, which gives a larger growth potential that of proprietary protocols [6]. There are several open solutions, like Contiki, TinyOS and FreeRTOS, which support various types of hardware, similarly to IEEE 802.15.4 and ZigBee stacks.

Contiki is a lightweight (e.g. $2 \mathrm{kB}$ of RAM and $40 \mathrm{kB}$ of application [7]) multi-tasking operating system. A support for both IPv6 and IPv4 is provided by uIP stack, which is a part of the project. TCP, UDP and ICMP protocols are supported, however without multicast functionality. In uIP stack mesh routing is currently not implemented. An alternative protocol stack which comes with Contiki is called Rime. It provides a range of communication primitives, which allow to implement and evaluate various mesh routing solutions and several other features. A modular structure of Contiki simplifies insertion of new functionalities [8]. Contrary to Contiki, TinyOS has monolithic architecture. Both multi-threading and event-driven programming model are supported. Components of systems are created by dedicated nesC language. Mesh routing with IPv6 is possible.

6LoWPAN is developing technology with several implementations. Features and constrains vary depending on applied operating systems and stack. A significant drawback in context of the TULCOEMPA is additional IP overhead, which might decrease available throughput. Despite that, it is worth to evaluate diverse communication standards, which will enable possibility to find the most effective solution for the discussed project. 


\section{F. Bluetooth Low Energy}

Bluetooth Low Energy (BLE) has been specified in Bluetooth version 4.0 [9]. This standard is completely different from earlier Bluetooth specifications and not backwardcompatible. The primary attribute toward other Bluetooth specifications is the fact that it is not connection-oriented, therefore no complex connection procedures are required. Essential improvement over IEEE 802.15.4 and ZigBee is adaptive frequency hopping spread spectrum collision avoidance mechanism, which efficiently confronts interferences by deflecting busy channels. Available spectrum has been divided into 40 channels, 37 of which are destined for data transmission, and the remaining three for advertising, discovery and initiations. In contrast to ZigBee, GFSK instead of OFDM modulation has been applied. Moreover, frequency hopping increases robustness and does not require waiting for the end of other devices transmissions. This reduces transceiver idle time, which significantly improves energy performance. BLE supports star topology only. There are defined two types of devices, master and slave. Master is able to manage multiple simultaneous connections with slave devices, while slave can be connected only to the master. Communication occurs by synchronous waking states, for the rest of the time slaves are in sleep mode. A lack of support for mesh topology is the main deficiency of BLE standard, however it is worth emphasizing, that energy cost for sending the same amount of data is lower than for 802.15 .4 [10]. Moreover, theoretical throughput of BLE is $1 \mathrm{Mb} / \mathrm{s}$, and for $802.15 .4,250 \mathrm{~kb} / \mathrm{s}$ only. Therefore it is worth to consider using Bluetooth Low Energy in applications, where network physical size is relatively small and mesh topology is not required. A larger throughput is desirable for measurements requiring an immense amount of samples. However, Bluetooth Low Energy standard defines maximum payload in a data packet of 27 bytes, while ZigBee allows 104 bytes. As a result, efficiency of data transfer of higher amounts of measurement results might be decreased due to unnecessary frame overhead. Bluetooth Low Energy standard defines profiles to describe device functionality. Connection can be only initiated by a slave node with advertisement functionality.

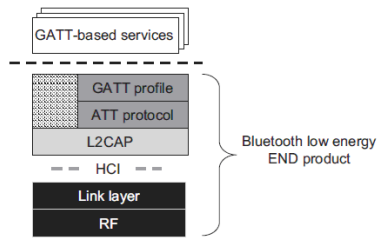

Fig. 5. Bluetooth low energy stack [3]

BLE stack is presented in Fig. 5. Similarly to older Bluetooth specifications, HCI (Host Controller Interface) defines API for link manager, which has a reduced command set. L2CAP (Link Layer Common Adaptation Layer) is responsible for providing interface layer, which is used by the protocol stack. L2CAP is also responsible for dividing larger packets to blocks with 27 byte of payload [3]. Therefore, using chip with only three first layers implemented could possibly enable increase in efficiency of sending higher amount of data by reducing unnecessary overhead. BLE, due to its higher throughput and low energy consumption appears to be an interesting choice for discussed project. Nevertheless, lack of the mesh topology support and small maximum payload size in a frame may be cogent issues.

\section{G. $D A S H 7$}

DASH7 is a trade name for ISO/IEC 18000-7 standard, which has been designed as active RFID. It is a constantly evolving solution developed by DASH7 alliance. ISO/IEC 18000-7 operates on $433 \mathrm{MHz}$ frequency. There are two modes available. Mode 1 is a simpler standard with only one channel available, which is $500 \mathrm{kHz}$ wide, with FSK modulation. Maximum possible throughput is $27.8 \mathrm{~kb} / \mathrm{s}$. Packets are synchronised by a pulse width. Collisions are avoided by using Slotted Aloha access method [11]. Because of the low throughput and inefficient bandwidth utilisation, DASH7 Mode 1 is not suitable solution for WSN in the TULCOEMPA project, despite a low power consumption related to its simplicity.

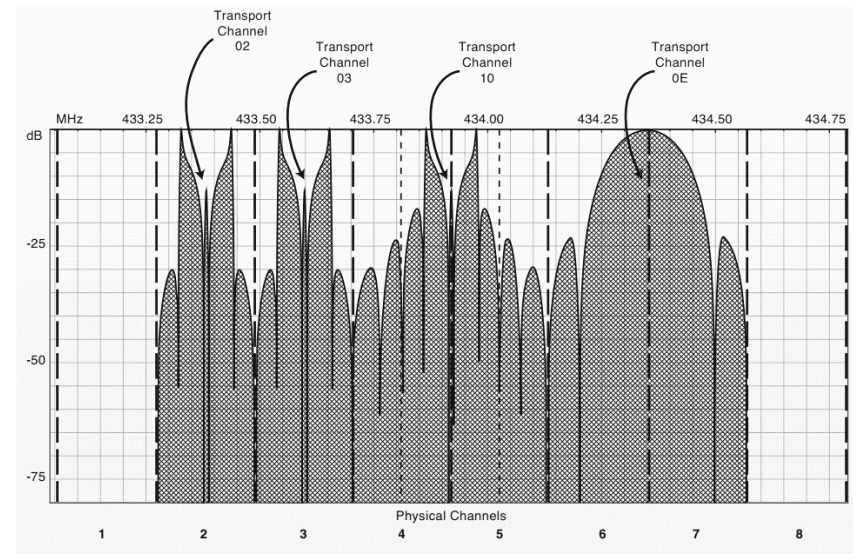

Fig. 6. Example of DASH7 channel assignment [11].

DASH7 Mode 2 provides throughput from $27.8 \mathrm{~kb} / \mathrm{s}$ to $200 \mathrm{~kb} / \mathrm{s}$, with GFSK modulation. There are 8 physical channels available, each of which is $216 \mathrm{kHz}$ wide. Physical channels might be bundled into a logical transport channel in order to increase available throughput. Sample channel configuration is presented in Fig. 6. Collision avoidance in DASH7 is handled by Carrier Sense Multiple Access. In opposite to standards like ZigBee, DASH7 requires no periodic network hand shaking or synchronisation between devices. Thus, receiver must be enabled to resolve a command from master and for instance, conduct a measurement procedure. MAC layer of DASH7 supports radio wakeup feature, which enables activation of the node by sending appropriate trigger signal, that can last up to 4.8 seconds. Handshake procedure is simplified in comparison to ZigBee, which leads to a decreased transmission time. DASH7 conducts request-response model, in other words, by default, only master can initiate a dialogue. As a result, a network created with DASH7 nodes is based on star topology. However, communication between nodes is possible. It could possibly allow to implement mesh routing in this technology. Although DASH7 is mainly destined for 
RFID tags, Mode 2 addresses wireless sensing applications, therefore it is possible to apply it for SHM solutions. DASH7 can be implemented on Texas Instruments CC430RF chip. OpenTag software, which is open source, implements MAC, PHY, Network and Transport OSI layers of DASH7 Mode 2. There are several other SoC transceivers supported. OpenTag is written in $\mathrm{C}$ and provides API, which enables addition of custom functionality. Size of the packet (called Universal Data Block) is not defined as a constant. It can be up to 255 bytes. Multi-frame packets are also supported [12]. When a significant amount of data is being sent, possibility for transmission interruption increases. It can be caused by external interferences. As a result, repetition of the frame could be required, causing unnecessary additional energy consumption. In comparison to other mentioned above communication standards, DASH7 is the simplest protocol, due to its RFID roots. A lack of mesh routing is significant disadvantage on larger objects, where even range provided by $433 \mathrm{MHz}$ band might be not satisfactory. Moreover, in particular scenarios, (e.g. dynamic strain measurements on a bridge) $200 \mathrm{~kb} / \mathrm{s}$ throughput can be too low. Despite mentioned disadvantages, DASH7 is suitable communication standards for e.g. static measurements of smaller structures.

\section{H. IEEE $802.11 n$}

IEEE 802.11 networks due to their protocol complexity, and as a result high computational requirements, are not able to operate for longer time periods on a battery power, which virtually restricts them to an external power supply. In comparison to various IEEE 802.15.4, DASH7, BLE and other standards, high throughput (up to $600 \mathrm{Mb} / \mathrm{s}$ ) is a substantial advantage. OFDM modulation is used. Channel width can be increased from 20 to $40 \mathrm{MHz}$ to enable higher data rate. Two bands are available, $2.4 \mathrm{GHz}$ and $5 \mathrm{GHz}$. Channel spacing is $5 \mathrm{MHz}$, therefore, in $2.4 \mathrm{GHz}$ band, to avoid overlapping and interference, only three channels $(1,6,11)$ should be used in one area. Most widely applied topology is a star, where nodes are connected with Access Point. This kind of network can be combined into extended service set. Node can move between Access Points with transparent routing, which enables connection maintenance. Ad-Hoc topology is also possible. Range of IEEE $802.11 \mathrm{n}$ has been increased by MIMO (Multiple Input, Multiple Output) technique. Multiple antennas on a device, for which there is dedicated radio circuit, enables forming a beam. As a result, directional EM emission characteristics is achieved. Moreover, resulting signal to noise ratio is higher. Additional benefit is reduced problem of multipath interference [3]. Because of complexity of the protocol and high power requirements, $\mathrm{WiFi}$ is not suitable communication solution for the discussed project. Despite that, the mentioned above technology can be complementary. As an example, may serve a wireless measurement network, where nodes send acquired data to a local coordinator using IEEE 802.15.4, which forwards results to a data centre using Wireless LAN. It is important to note that there were successful attempts of sensor networks with mesh topology using modified WiFi routers working under Linux [13]. Redpine Signals company offers module dedicated for WSN -
RS9110-N-11-03 [14]. It supports IEEE 802.11n both 2.4 and $5 \mathrm{GHz}$ bands. Available throughput is up to $65 \mathrm{Mb} / \mathrm{s}$. With $1 \mathrm{Mb} / \mathrm{s}$ data rate and TCP transmission, current consumption is estimated to $23 \mathrm{~mA}$, when $22 \mathrm{Mb} / \mathrm{s}-174 \mathrm{~mA}$ (for $3.3 \mathrm{~V}$ DC supply), which might be satisfactory accomplishment for particular applications. Awake time is significantly increased, because of the fact that connecting to a network after node awakening is a complex procedure. Therefore, IEEE $802.11 \mathrm{n}$ is not a suitable solution for the discussed project, due to complexity of the protocol, particularly if mesh routing is required.

\section{Weightless}

Weightless is an emerging standard for M2M communication. It utilizes "White Space" spectrum, which is an unused portion of TV broadcast band. In the USA it is possible to transmit on White Spaces without license, however a device has to meet requirements of FCC (Federal Communications Commission). In several European countries in a near future it will be also allowed to use Weightless devices without additional permissions. [15] Typical cell should support up to one million devices, routing between cells should be possible. Terminal output power should be up to $40 \mathrm{~mW}$, while a base station can transmit with $4 \mathrm{~W}$ [16]. High output power of base station will allow to apply less sensitive receiver circuit in Weightless node. In this way, a cost of a transceiver will be lower. Due to the presented architecture, mesh routing is not possible, only a star topology with roaming will be available. Throughput will vary depending on a distance from base station, might be up to $16 \mathrm{Mb} / \mathrm{s}$, but significantly decreasing with distance, even down to $0.0025 \mathrm{Mb} / \mathrm{s}$. The expected range is up to $20 \mathrm{~km}$. It can be achieved by DBPSK modulation scheme [17]. Transceivers are supposed to have a low energy consumption and relatively simple structure, to reduce cost of a chip. The first commercial release of the chip is expected around third quarter of 2013 .

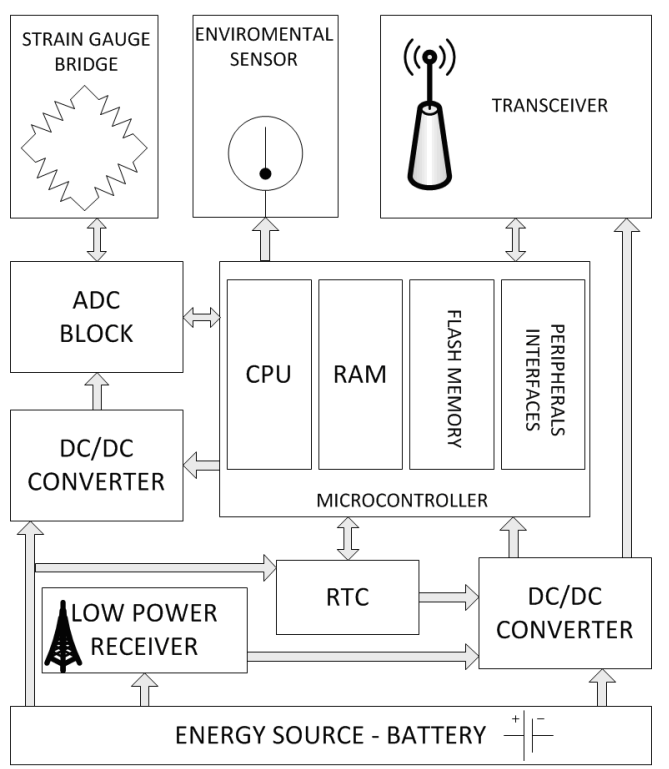

Fig. 7. Proposed WSN node structure for the TULCOEMPA project. 


\section{WirElESS StRAin SENSOR PROTOTYPE}

In the summary of the previous chapter, IEEE 802.15.4 and DASH7 offers only $250 \mathrm{~kb} / \mathrm{s}$, which might be not enough for dynamic measurements. IEEE 802.15.4a offers higher data rate, but availability of transceivers for this standard is not satisfactory. First two OSI layers of BLE appear to be interesting choice, if mesh routing and higher payload size would be implemented. Currently no open source stacks for BLE are available. The described project solution bases on IEEE 802.15.4, because of simplicity of migration to IEEE 802.15.4a in future. Moreover, it is a convenient platform to conduct tests with different topologies and routing solutions, e.g. Contiki with IPv6. On the other side, simplicity of DASH7 would allow to deploy an energy-efficient system, provided that it is possible to analyse the data and compress them locally, to fit possible data rate constrains.

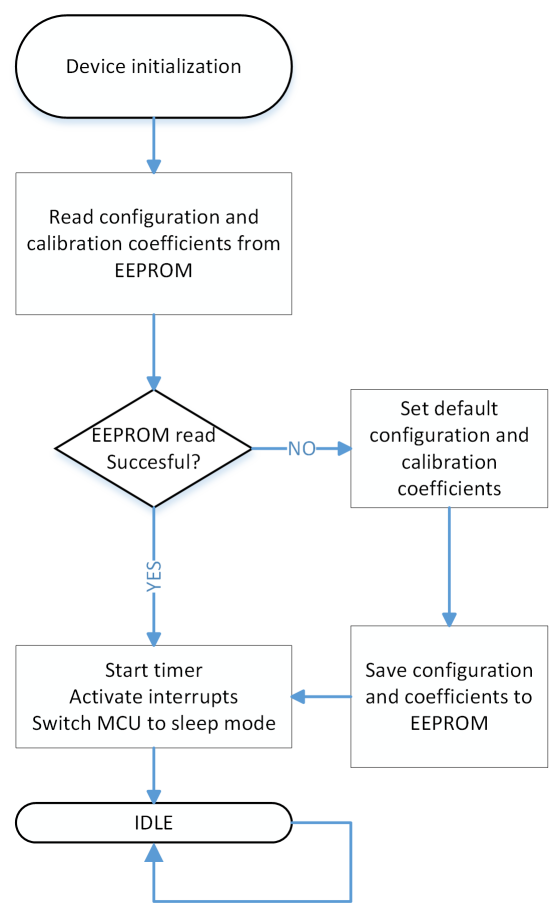

Fig. 8. Structure of the main program.

The important part of system design is a way of awakening WSN nodes by external trigger from Truck Recognition System (TRS). The receiver should be able to listen with as small power consumption as possible, to preserve device energy. In this case DASH7 is the most efficient. For IEEE 802.15.4 it might be worth to consider an additional simple low power receiver, if LPL (Low Power Listening) techniques were not efficient enough. Alternative solution is an activation of dynamic measurements, when a value acquired from low frequency measurement will be alarming. Nonetheless, many short-time strain changes and its initial value will be omitted, which makes this solution less suitable for the TULCOEMPA project.

Fig. 7 presents the proposed block structure of measurement node. Low power receiver and Real Time Clock (RTC) are connected to a power source, e.g. battery. The strain gauge

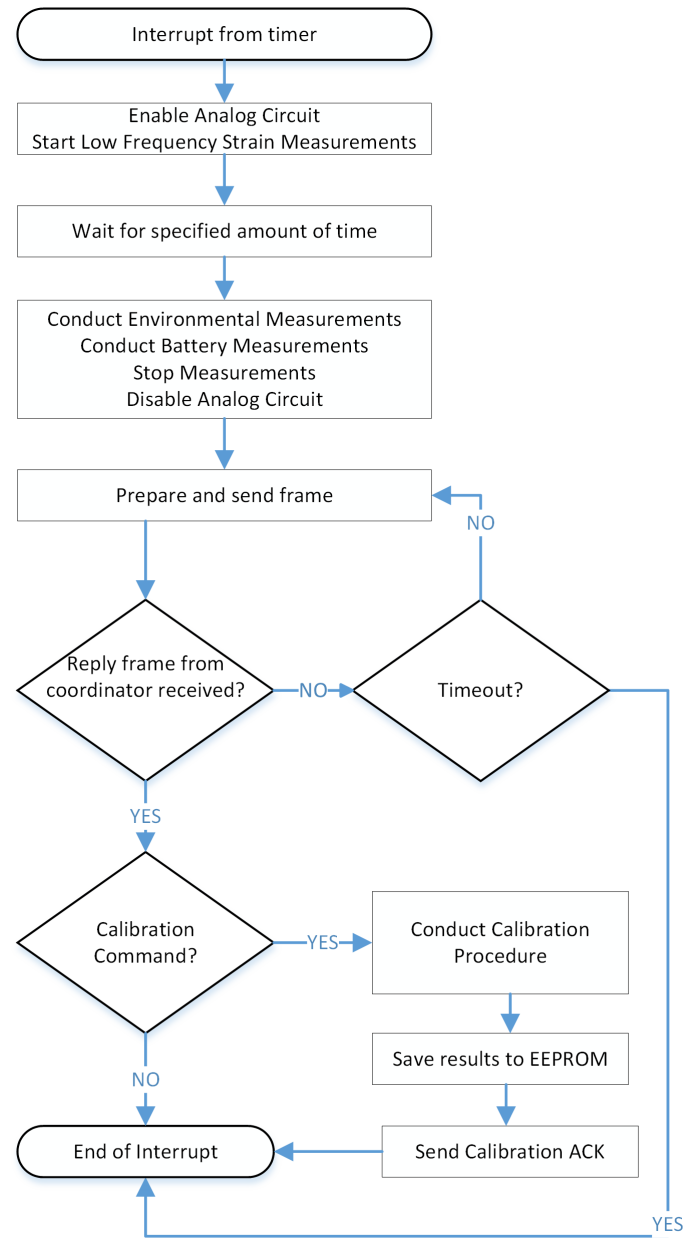

Fig. 9. Structure of the interruption handler program.

bridge and ADC (Analog-Digital Converter) have separate DC/DC power converter, which is activated by microcontroller for the shortest possible time. Microcontroller, transceiver and other parts have their own power converter, which can be enabled by RTC and low power receiver. This enables node activation only at a specified hour or by TRS trigger. Strain gauges are connected to $\mathrm{ADC}$, which provides both excitation voltage on bridge and output voltage measurement. When microcontroller is woken up, it enables analogue circuit and starts strain measurement. During this activity, environmental conditions are also monitored. When ADC sets interruption flag, microcontroller reads data from ADC and disables analogue circuit. After computation of acquired values, the microcontroller may enable transceiver to send results to a network gate and further to a remote server.

Fig. 10 presents early WSN prototype. Temperature, humidity and strain measurements have been implemented. The device sends results to a network coordinator, which acquires measured data. Voltage on the electrical strain gauge bridge is excited with $5 \mathrm{~V}$ amplitude. As an ADC, the Analog Devices AD7730 has been applied. It is 24-bit delta-sigma analog-digital converter with SPI (Serial Peripheral Interface) and auto calibration functionality. The supported sampling rate is up $1200 \mathrm{~Hz}$, which is satisfactory for most cases of 
strain measurements. The programmable gain and offset are stable and temperature changes have insignificant influence on them. Input is protected from ESD and other over-voltage by transient-voltage-suppression (TVS) diodes connected to the every line of input. For temperature and humidity, Sensirion SHT-11 sensor has been applied, because of its digital serial interface and pre-calibration conducted by manufacturer. For the microcontroller and transceiver, integrated module ATZB24-UFL from Atmel has been used. It consists of AVR ATmega 1281 8-bit microcontroller and IEEE 802.15.4 compliant AT86230RF transceiver. The applied MCU (Microcontroller Unit) has peripherals and computing capabilities enabling measurement conduction and sending results to the coordinator of the network. At this state, the measurement program and stack are working on the same microcontroller. Therefore, several constraints might occur. In future, separate MCU for protocol stack will be considered. For IEEE 802.15.4 stack implementation, $\mu$ racoli [18] has been used. Communication is performed on $2.4 \mathrm{GHz}$ band, with $100 \mathrm{~mW}$ output power.

Fig. 8 presents behaviour of the device after powering up. At first, hardware is initialized and checked. Subsequently, MCU attempts to read configuration and calibration coefficients, which concern strain measurements, from EEPROM memory. If a required information is not found, defaults are being set, and a coordinator will be informed about the problem on the next communication occurrence. The following step of the program is to activate internal timer and the MCU interrupts, then the device is being switched into a sleep mode. When interrupt from timer occurs, procedure from Fig. 9 is being executed. The device enables analogue circuit and performs strain measurements for a defined amount of time. This enables acquisition of an average value. During this activity, environmental conditions and voltage on module battery might be also monitored. When the procedure is finished, the analogue circuit is disabled to reduce energy consumption. At the next step, a data frame is being prepared and sent to the coordinator. When the coordinator does not reply, the module repeats transmission for a specified period of time or number of attempts. When reply frame consists of a calibration command, calibration of the strain measurement circuit is performed. At the end of calibration procedure, acknowledgement is sent to the coordinator. Afterwards, the device switches to a sleep mode without waiting for confirmation from the coordinator.

The measurement module is powered by two DC-DC converters, MCP1640 from Microchip. Switching frequency of $500 \mathrm{kHz}$ reduces possibility of interference between the power circuit and the measurement circuit. The enable PIN allows to switch power off, when a measurement is not performed. The digital part is powered with $3.3 \mathrm{~V}$ and the analogue one with $5 \mathrm{~V}$. The module is equipped with two AA batteries.

\section{CONCLUSION}

This paper describes the design process of the wireless, distributed strain measurement system for the TULCOEMPA project. The proper choice of a radio band has been discussed and the wireless sensor network architecture proposed. The

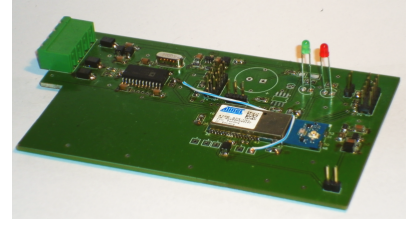

Fig. 10. Early wireless strain measurement device

presented chapters describe several available communication standards, its advantages, issues, areas of possible deployment. Finally, the prototype of the solution has been presented. Architecture of the system, reasons for the chosen components, measurement and communication algorithms have been described. The proposed design enables introduction of several enhancements in future.

\section{ACKNOWLEDGMENT}

The authors would like to thank all members of the TULCOEMPA project, from KBB, EMPA and DMCS.

\section{REFERENCES}

[1] (2013, Feb.) Tulcoempa project homepage. [Online]. Available: http://www.tulcoempa.com

[2] A. Willig, "Wireless sensor networks: concept, challenges and approaches," Elektrotechnik und Informationstechnik, vol. 123, pp. 224-231, 2006. [Online]. Available: http://dx.doi.org/10.1007/s00502006-0351-1

[3] N. Hunn, Essentials of Short-Range Wireless, ser. The Cambridge Wireless Essentials Series. Cambridge University Press, 2010. [Online] Available: http://books.google.pl/books?id=pSKudsi $\backslash$ kWEC

[4] (2013, Feb.) IEEE 802.15 WPAN Task Group 4 (TG4. [Online]. Available: http://www.ieee802.org/15/pub/TG4.html

[5] E. Karapistoli, F.-N. Pavlidou, I. Gragopoulos, and I. Tsetsinas, "An overview of the ieee 802.15.4a standard," Communications Magazine, $I E E E$, vol. 48 , no. 1 , pp. 47-53, january 2010.

[6] N. Kushalnagar, G. Montenegro, and C. Schumacher, "IPv6 over Low-Power Wireless Personal Area Networks (6LoWPANs): Overview, Assumptions, Problem Statement, and Goals," RFC 4919 (Informational), Internet Engineering Task Force, August 2007. [Online]. Available: http://www.ietf.org/rfc/rfc4919.txt

[7] (2013, Feb.) The Contiki Operating System. [Online]. Available: http://contiki.sourceforge.net/docs/2.6

[8] M. O. Farooq and T. Kunz, "Operating systems for wireless sensor networks: A survey," Sensors, vol. 11, no. 6, pp. 5900-5930, 2011. [Online]. Available: http://www.mdpi.com/1424-8220/11/6/5900

[9] (2010, Jun.) BLUETOOTH SPECIFICATION version 4.0 [VOL 0]. [Online]. Available: https://www.bluetooth.org/Technical/Specifications/ adopted.htm

[10] M. Siekkinen, M. Hiienkari, J. Nurminen, and J. Nieminen, "How low energy is bluetooth low energy? comparative measurements with zigbee/802.15.4," in Wireless Communications and Networking Conference Workshops (WCNCW), 2012 IEEE, april 2012, pp. 232-237.

[11] J. Norair. (2010, aug) Mode 2 Webminar. [Online]. Available: https://dash7.memberclicks.net/assets/PDF/mode2\%20webinar.pdf

[12] "ISO 18000-7 Mode 2 Specification," 2011, draft 012

[13] M. Scheidgen, A. Zubow, and R. Sombrutzki, "HWL - A high performance wireless sensor research network," in Networked Sensing System. (INSS), 2012 Ninth International Conference on, june 2012, pp. 1-4.

[14] (2013, Feb.) Redpine Signals. [Online]. Available: http://www. redpinesignals.us/

[15] N. Mouyal, "Using spectrum 'white spaces' in europe," DigiTAG, November 2012.

[16] Weightless, "Weightless Specification Overview," 2012.

[17] W. Webb, Understanding Weightless: Technology, Equipment, and Network Deployment for M2M Communications in White Space. Cambridge University Press, 2012. [Online]. Available: http://books. google.pl/books?id=eOJ4fZYG2WAC

[18] (2013, Feb.) The uController Radio Communication Library. [Online] Available: http://www.nongnu.org/uracoli/ 